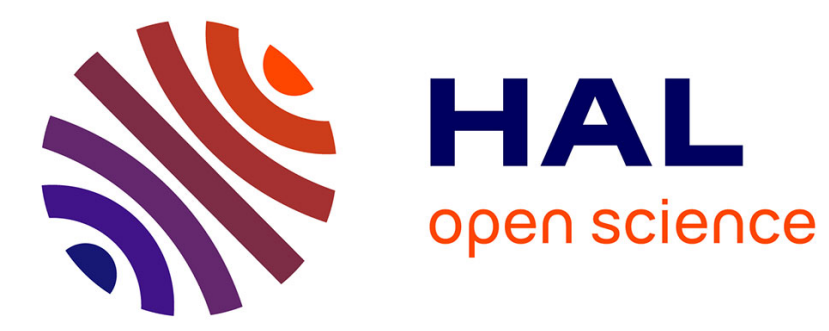

\title{
Etude des écoulements permanents en conduite cylindrique, d'une suspension, à partir des données des spectres Doppler obtenus en débitmétrie ultrasonore
}

\author{
J.C. Vera, J.P. L'Huillier
}

\section{To cite this version:}

J.C. Vera, J.P. L'Huillier. Etude des écoulements permanents en conduite cylindrique, d'une suspension, à partir des données des spectres Doppler obtenus en débitmétrie ultrasonore. Revue de Physique Appliquée, 1980, 15 (8), pp.1347-1355. 10.1051/rphysap:019800015080134700 . jpa-00244858

HAL Id: jpa-00244858

https://hal.science/jpa-00244858

Submitted on 1 Jan 1980

HAL is a multi-disciplinary open access archive for the deposit and dissemination of scientific research documents, whether they are published or not. The documents may come from teaching and research institutions in France or abroad, or from public or private research centers.
L'archive ouverte pluridisciplinaire HAL, est destinée au dépôt et à la diffusion de documents scientifiques de niveau recherche, publiés ou non, émanant des établissements d'enseignement et de recherche français ou étrangers, des laboratoires publics ou privés. 


\title{
Etude des écoulements permanents en conduite cylindrique, d'une suspension, à partir des données des spectres Doppler obtenus en débitmétrie ultrasonore
}

\author{
J. C. Vera $(*)$ et J. P. L’Huillier \\ Institut de Génie Biologique et Médical, 24, rue Lionnois, 54000 Nancy, France
}

(Reçu le 7 janvier 1980, révisé le I"1 avril 1980, accepté le 28 avril 1980)

\begin{abstract}
Résumé. - L'analyse spectrale basses fréquences des signaux Doppler obtenus en débitmétrie ultrasonore est utilisée pour l'étude des écoulements permanents d'une suspension, en conduite cylindrique. Dans ce travail, nous présentons d'abord une brève approche théorique des spectres, dans le cas des écoulements laminaires et turbulents, prenant en compte l'influence du profil des vitesses pour différentes lois de concentration en particules. L'exploitation des données spectrales enregistrées est réalisée à partir des modèles que nous avons développés. Il est alors possible de déduire de ces représentations, les paramètres énergétiques et hydrodynamiques des écoulements étudiés (énergie du signal de réponse Doppler de l'écoulement ; vitesse moyenne ou nombre de Reynolds).

Abstract. - The spectral analysis, in low frequencies, of spectra obtained in ultrasonic debitmetry is used for study of permanent flows, in cylindrical pipe, of a fluid containing particles. In this work firstly present brievely theoretical mathematical approach of these spectra for laminar and turbulent flows, showing the influence of the velocity profile and the particle concentration in the flows on the shape of the Doppler spectra. The spectral data are analysed with the use of the mathematical models developed from what we know about the characteristics of the spectra. We are then able to obtain the energetic and hydrodynamic parameters of the studied flows (energy of the Doppler signal of the flow ; average velocity or Reynolds number).
\end{abstract}

1. Introduction. - Lorsqu'une onde plane ultrasonore, produite par une source émettrice, se propage dans une suspension en mouvement dans une conduite cylindrique, sa réflexion ne s'effectue pas sur une cible unique et stable mais sur un ensemble de cibles discrètes et fluctuantes. Ainsi, chacune d'elles peut être considérée comme une source d'ondes sphériques (taille des cibles faible devant la longueur d'onde) qui vont se propager et être en partie captées par le récepteur. Celui-ci ne reçoit donc pas une fréquence unique mais tout un spectre de fréquences qui résulte de la composition, au niveau du récepteur, des ondes sphériques élémentaires renvoyées par chaque particule en mouvement avec le fluide ([1], [2]).

Ainsi, dans le cas de l'écoulement d'un fluide contenant un grand nombre de particules, il se produit un élargissement du spectre de raie obtenu dans le cas d'une particule unique ([3], [4]). Le spectre d'amplitude obtenu est représentatif de l'écoulement étudié (Fig. 1)

$\left(^{*}\right)$ Laboratoire d'Energétique et de Mécanique Théorique et Appliquée (LEMTA, ERA 595), Ecole des Mines, Parc de Saurupt, 54000 Nancy, France. et son tracé varie avec les conditions qui régissent cet écoulement.

Par conséquent, de la connaissance et de l'étude d'un tel spectre, il est possible de remonter jusqu'aux caractéristiques de l'écoulement (nombre de Reynolds; énergie du signal de réponse Doppler) indépendam-

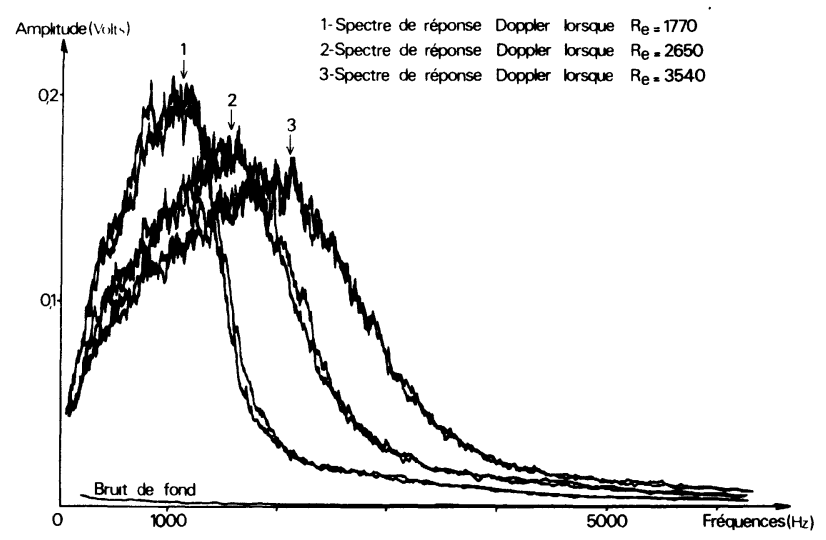

Fig. 1. - Spectres de réponse Doppler correspondant à différentes valeurs du nombre de Reynolds.

[Doppler spectra obtained for several Reynolds numbers.] 
ment de son régime. L'analyse spectrale basses fréquences, en temps réel, du signal Doppler est une des méthodes d'investigation que nous avons envisagée dans nos travaux sur l'étude de l'écoulement sanguin ([5], [6]). Dans ce travail nous proposons, après avoir présenté brièvement quelques études théoriques de l'influence du profil de vitesses sur le spectre d'amplitude de réponse Doppler d'un écoulement permanent, une modélisation des spectres d'amplitude expérimentaux permettant l'exploitation des résultats. Contrairement à certains travaux qui font appel à une étude trop restrictive du phénomène ([7], [8]), cette modélisation ne fait appel à aucune hypothèse sur la diffusion des ondes ultrasonores dans un milieu dispersif. Elle consiste à faire le choix d'une fonction mathématique de répartition spectrale et à ajuster ses paramètres afin que sa représentation se confonde avec le graphe des spectres expérimentaux.

2. Etude d'un écho. - Soit $X_{\mathrm{E}}(t)=G_{\mathrm{E}} \cdot \cos \left(2 \pi v_{\mathrm{E}} t\right)$ le signal d'émission. L'onde ultrasonore incidente qui lui correspond est diffusée par chaque particule se trouvant à l'intérieur du volume fluide intercepté par le faisceau incident. Cette onde, qui subit une altération de fréquence (effet Doppler), du fait du mouvement des particules, est partiellement réfléchie vers le récepteur.

Compte tenu de la faible dimension des particules par rapport à la longueur d'onde du signal, nous admettons que la diffusion se fait de façon homogène et isotrope.

Analysons le phénomène élémentaire. Soit $S_{i}(t)$ le signal capté par le récepteur et provenant de la réflexion du signal incident par la particule $i$ (Fig. 2) :

$$
S_{i}(t)=G\left(v_{\mathrm{R} i}\right) \cdot \cos \left[2 \cdot \pi \cdot v_{\mathrm{R} i} \cdot t+\varphi\left(v_{\mathrm{R} i}\right)\right]
$$

expression dans laquelle $v_{\mathrm{R} i}$ est la fréquence de l'onde diffusée et $\varphi\left(v_{R i}\right)$ la phase qui dépend de la distance émetteur-particules-récepteur, donc indirectement de la fréquence $v_{F}$ qui est reliée à la fréquence de l'onde incidente par la relation :

$$
v_{\mathrm{R} i}=r_{i} \cdot v_{\mathrm{E}}
$$

avec

$$
r_{i}=1-\frac{V_{i}}{C}\left(\cos \theta_{\mathrm{R} i}-\cos \theta_{\mathrm{E} i}\right) .
$$

La grandeur $G\left(v_{\mathbb{R} i}\right)$ est fonction, en première approximation :

- de l'amplitude de l'onde incidente

- de l'affaiblissement de l'onde ultrasonore avec la distance parcourue.

Si nous considérons le volume fluide qui reçoit les ultrasons, et que nous appellerons volume insonifié ; les particules sont au nombre de $N$; la représentation mathématique du signal capté par le récepteur est de la forme

$$
\begin{aligned}
& X_{\mathrm{R}}(t)=\sum_{i=1}^{N} S_{i}(t) \\
& X_{\mathrm{R}}(t)=\sum_{i=1}^{N} G\left(v_{\mathrm{R} i}\right) \cdot \cos \left[2 \pi \cdot v_{\mathrm{R} i} \cdot t+\varphi\left(v_{\mathrm{R} i}\right)\right] .
\end{aligned}
$$

$\mathrm{Du}$ fait de la grande population de particules présentes dans le volume insonifié, nous pouvons assimiler leur distribution discrète à une distribution continue, ce qui nous amène à considérer également
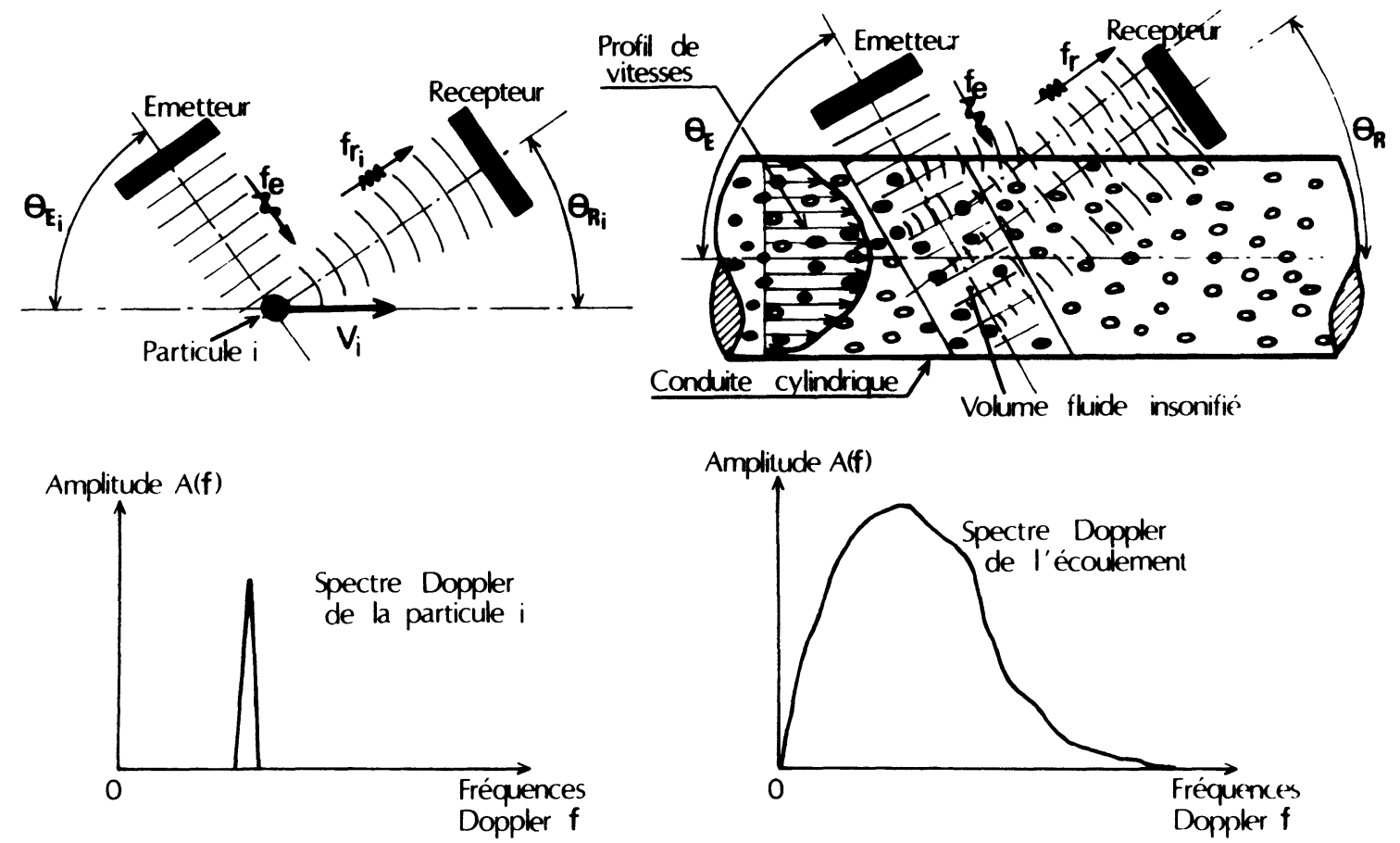

Fig. 2. - Definition du volume fluide insonifié et forme des spectres d'amplitude de réponse Doppler. 
une distribution continue des fréquences $v_{\mathrm{R} i}$. Si nous appelons $\rho\left(v_{\mathrm{R} i}\right)$ la fonction de distribution de ces fréquences, la relation devient :

$$
\begin{gathered}
X_{\mathrm{R}}(t)=\int_{0}^{+\infty} \rho\left(v_{\mathrm{R}}\right) \cdot G\left(v_{\mathrm{R}}\right) \cdot \cos \left[2 \pi \cdot v_{\mathrm{R}} \cdot t+\varphi\left(v_{\mathrm{R}}\right)\right] \cdot \mathrm{d} v_{\mathrm{R}} . \\
\text { Posons } M\left(v_{\mathrm{R}}\right)=\rho\left(v_{\mathrm{R}}\right) \cdot G\left(v_{\mathrm{R}}\right) \\
X_{\mathrm{R}}(t)=\int_{0}^{+\infty} M\left(v_{\mathrm{R}}\right) \cdot \cos \left[2 \pi \cdot v_{\mathrm{R}} \cdot t+\varphi\left(v_{\mathrm{R}}\right)\right] \cdot \mathrm{d} v_{\mathrm{R}}
\end{gathered}
$$

$M\left(v_{\mathrm{R}}\right)$ est positif et représente l'amplitude de chaque sinusoïde qui comporte le signal haute fréquence capté par le récepteur, et $\varphi\left(v_{\mathbf{R}}\right)$ est leur phase.

2.1 APPROChe mathématiQue DU SIGNAL DopPLER. - Afin d'obtenir la composante Doppler du signal capté par le récepteur nous mélangerons, par multiplication, le signal d'émission et le signal de réception :

$$
\begin{gathered}
S(t)=\left\{G_{\mathrm{E}} \cdot \cos 2 \pi v_{\mathrm{E}} \cdot t\right\} *\left\{\int_{0}^{+\infty} M\left(v_{\mathrm{R}}\right) \cdot \cos \left[2 \pi \cdot v_{\mathrm{R}} \cdot t+\varphi\left(v_{\mathrm{R}}\right)\right] \cdot \mathrm{d} v_{\mathrm{R}}\right\} \\
\begin{array}{r}
S(t)=G_{\mathrm{E}} \cdot \int_{0}^{+\infty} M\left(v_{\mathrm{R}}\right) \cdot \cos \left[2 \pi\left(v_{\mathrm{E}}+v_{\mathrm{R}}\right) \cdot t+\varphi\left(v_{\mathrm{R}}\right)\right] \cdot \mathrm{d} v_{\mathrm{R}}+ \\
+G_{\mathrm{E}} \cdot \int_{0}^{+\infty} M\left(v_{\mathrm{R}}\right) \cdot \cos \left[2 \pi\left(v_{\mathrm{R}}-v_{\mathrm{E}}\right) \cdot t+\varphi\left(v_{\mathrm{R}}\right)\right] \cdot \mathrm{d} v_{\mathrm{R}} .
\end{array}
\end{gathered}
$$

Au moyen d'un filtre supposé idéal, nous ne conservons que les composantes basses fréquences, $v=v_{\mathrm{R}}-v_{\mathrm{E}}$, du signal $S(t)$. Nous obtenons :

$$
S_{\mathrm{F}}(t)=\int_{0}^{+\infty} G(v) \cdot \cos [2 \pi \cdot v \cdot t+\Psi(v)] \cdot \mathrm{d} v
$$

où $G(v)$ représente l'amplitude de chaque sinusoïde qui compose le signal basse fréquence $S_{\mathrm{F}}(t)$, c'est-à-dire son spectre d'amplitude, et $\Psi(v)$ leur phase.

Remarques. - La représentation mathématique que nous donnons du signal Doppler permet d'établir une certaine analogie avec le bruit Gaussien de valeur moyenne nulle. Nous savons, en effet, qu'un tel bruit est composé d'un grand nombre de sinusoïdes de fréquences différentes et de phases tout à fait aléatoires [9]. L'écriture mathématique d'un tel bruit est alors :

$$
n(t)=\int_{0}^{+\infty} N(v) \cdot \cos [2 \pi \cdot v \cdot t+\zeta(v)] \cdot \mathrm{d} v .
$$

2.2 INTÉRÊT DE LA CONNAISSANCE DU SPECTRE D'AMPLITUDE POUR NOTRE ÉTUDE. - La connaissance du spectre d'amplitude $G(v)$ conduit à une relation avec la vitesse moyenne de l'écoulement, soit d'après Roevros [10] :

$$
\bar{v}=\alpha \cdot \bar{V}=\frac{\int_{0}^{+\infty} v \cdot G^{2}(v) \cdot \mathrm{d} v}{\int_{0}^{+\infty} G^{2}(v) \cdot \mathrm{d} v}
$$

Cette relation suppose que chaque particule apporte la même contribution en puissance $\varphi_{0}$ au spectre (effet de masque, entre particules, négligeable).
3. Représentation analytique du spectre $G(v)$. Plusieurs théories tendent vers une approche analytique de ce spectre. La première méthode consiste à choisir une représentation statistique adaptée à la modélisation de la diffusion des ondes ultrasonores, de façon à ce que les spectres obtenus par le calcul coïncident avec les spectres expérimentaux. Malheureusement les hypothèses de départ, obligatoirement restrictives, conduisent à des résultats difficilement interprétables [7]. Une autre approche est tentée par Roevros qui introduit des hypothèses restrictives quant au phénomène de diffusion des ondes à l'intérieur du volume fluide insonifié [10].

En admettant, des lois de concentration particulaires approchées, de la forme

$$
C(r)=C_{\max }\left[1-\left(r / R_{0}\right)^{2 n}\right]
$$

l'amplitude du spectre $G(v)$ s'écrit, d'après cet auteur :

$$
G(v)=\frac{2 \pi \cdot d \cdot C_{\max }}{\alpha \cdot \sin \theta_{\mathrm{E}}} \cdot \varphi_{0} \cdot\left[1-\left(r / R_{0}\right)^{2 n}\right] \cdot r \cdot \frac{\mathrm{d} r}{\mathrm{~d} V} .
$$

Dans le cas d'un régime laminaire à l'intérieur du tube, la répartition des vitesses suit la loi parabolique connue [11] :

$$
\frac{V}{V_{\max }}=1-\left(\frac{r}{R_{0}}\right)^{2}
$$

et l'expression de l'enveloppe du spectre devient :

$$
G(v)=G_{0}\left[1-\left(1-V / V_{\max }\right)^{n}\right]
$$

avec $G_{0}=\left(\pi \cdot d \cdot R_{0}^{2} \cdot C_{\max } \cdot \varphi_{0}\right) /\left(v_{\max } \cdot \sin \theta_{\mathrm{E}}\right)$ qui représente la puissance du signal Doppler correspondant à une concentration particulaire uniforme égale à $C_{\max }$. 
La - figure $3 a$ montre des exemples de courbes $G(v)$ obtenues pour différentes lois $C(r)$ correspondant aux valeurs du paramètre $n=0 ; 1 / 2 ; 2 ; 5$.

Dans le cas du régime turbulent, on peut utiliser la loi de distribution de vitesse de la forme [11] :

$$
\frac{V}{V_{\max }}=\left[1-\left(\frac{r}{R_{0}}\right)\right]^{1 / 7} \text {. }
$$

Ce qui donne pour $G(v)$ :

$$
\begin{aligned}
& G(v)=14 G_{0}\left(\frac{v}{v_{\max }}\right)^{6} {\left[1-\left(\frac{v}{v_{\max }}\right)^{7}\right] \times } \\
& \times\left\{1-\left[1-\left(\frac{v}{v_{\max }}\right)^{7}\right]^{2 n}\right\}
\end{aligned}
$$
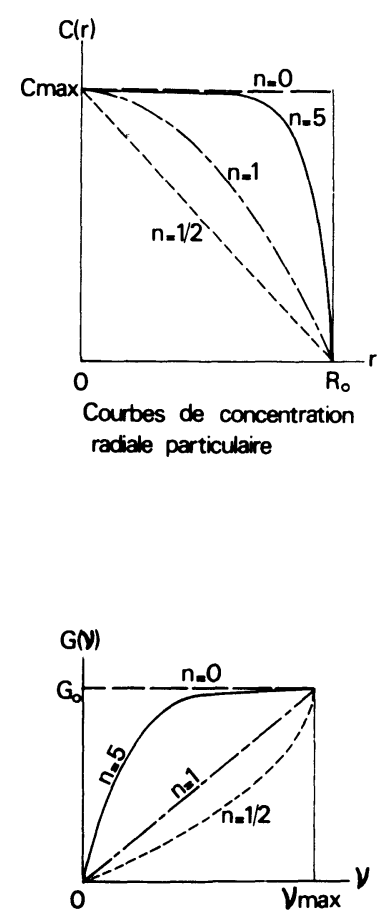

a. Spectre de réponse Doppler de lécoulement laminaire

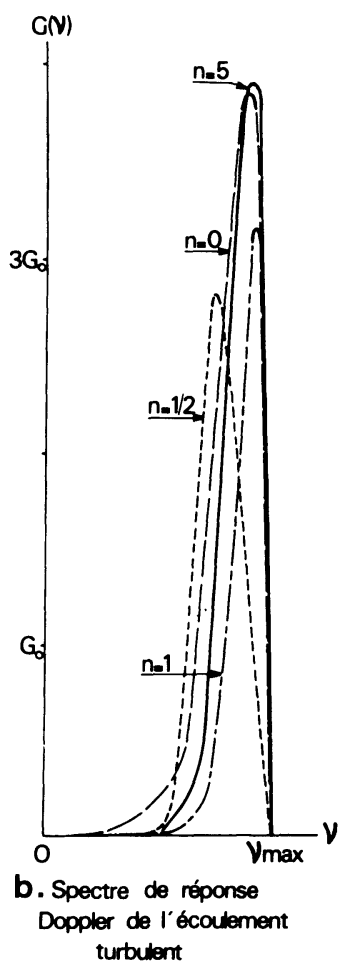

Fig. 3. - Spectres de densité de puissance de réponse Doppler $G$-v, pour différentes valeurs de $n: a$ ) écoulement laminaire; $b$ ) écoulement turbulent.

[Theoretical Doppler spectra corresponding to permanent Flow : a) laminar; $b$ ) turbulent.]

La figure $3 b$ représente les spectres $G(v)$ pour les mêmes valeurs du paramètre $n$.

La comparaison des figures $3 a$ et $3 b$ indique que dans le cas des écoulements turbulents, contrairement aux écoulements laminaires où le spectre est plus étalé, toute la puissance du signal Doppler est concentrée dans une bande de fréquences qui s'apparente à un spectre de raie. Cette forme de spectre s'explique en remarquant que la répartition dite en $1 / 7$ correspond à un profil des vitesses pratiquement plat et que les particules diffusent l'énergie ultrasonore incidente en provoquant sensiblement la même déviation Doppler $v_{\max } \# \alpha \cdot V_{\max }$.

4. Description du dispositif expérimental. - 4.1 BANC HYDRODYNAMIQUE. - La figure 4 représente le banc de mesure hydrodynamique permettant d'effectuer les mesures ultrasonores de vitesse moyenne, en écoulement permanent. Les caractéristiques du fluide sont connues et fixées : la suspension que nous utilisons comprend une phase liquide $(45 \%$ d'eau et $55 \%$ de glycérine; densité $\left.\rho_{\mathrm{F}}=1,17\right)$ et une phase solide (particules d'amidon, de forme sensiblement sphérique, dont la taille moyenne est de quelque $30 \mu \mathrm{m}$ et la concentration de $30 \mathrm{~g} / 1$; densité $\rho_{\mathrm{P}}=1,21$ ). La viscosité cinématique de cette suspension, à $37^{\circ} \mathrm{C}$, est $v_{\mathrm{c}}=0,04 \mathrm{St}$.

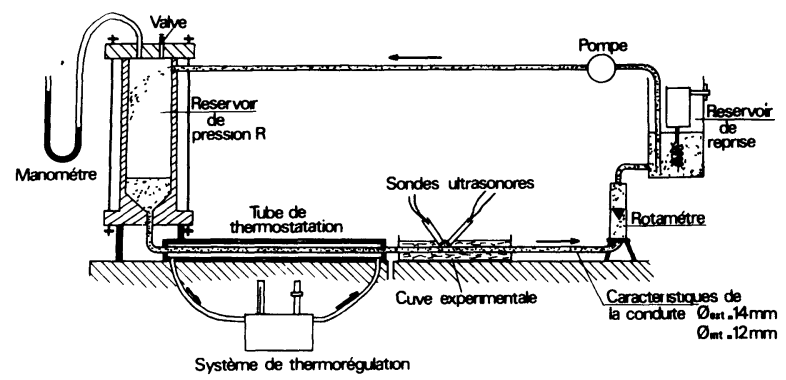

Fig. 4. - Banc hydrodynamique expérimental.

[Hydrodynamic bench.]

4.2 DéteCtion et anAlyse DU Signal. - L'appareil que nous utilisons est un débitmètre ultrasonore (Parks Electronics, modèle 806) directionnel, à émission continue, dont la fréquence de travail est de $10 \mathrm{MHz}$. La figure 5 représente le schéma synoptique de sa partie détection. Le signal Doppler obtenu est appliqué à l'entrée d'un analyseur (Hewlett Packard, modèle $8556 \mathrm{~A}$ ). Le spectre d'amplitude de réponse Doppler de l'écoulement étudié est alors visualisé sur l'écran mémoire de l'analyseur puis enregistré sur table traçante afin d'en permettre une étude quantitative. Un voltmètre R.m.s, adjoint à cette chaîne,

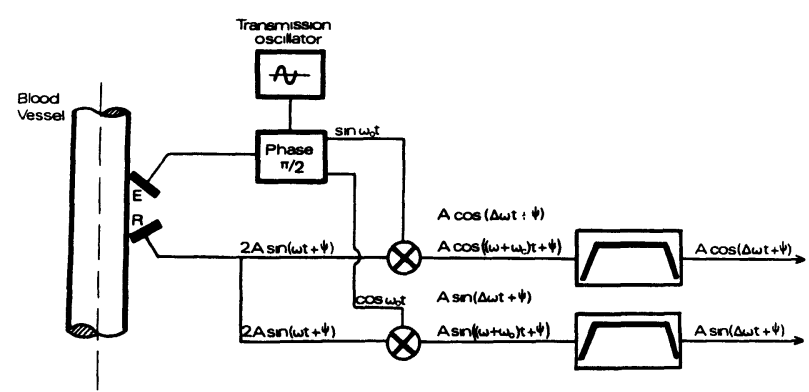

Fig. 5. - Synoptique du système de détection du signal de réponse Doppler de l'écoulement.

[Bloch diagram of the detection system.] 
permet d'obtenir une indication sur la puissance du signal de réponse Doppler.

Les deux sondes, émettrice et réceptrice, sont constituées de céramiques piézoélectriques en forme de disques circulaires, de $10 \mathrm{~mm}$ de diamètre, polarisés axialement et vibrant en épaisseur. L'orientation des sondes est repérée, par rapport à la direction de l'écoulement, par les angles $\theta_{\mathrm{E}}$ et $\theta_{\mathbf{R}}$. Ces angles sont pris respectivement égaux à $70^{\circ}$ et $55^{\circ}$, valeurs qui correspondent au maximum de puissance du signal de réponse Doppler de l'écoulement [5], [12].

4.3 CARACTÉRISTIQUES DES ÉCOULEMENTS ÉTUDIÉS. - Nous nous sommes attachés à l'étude d'écoulements permanents dont les nombres de Reynolds correspondent aux domaines des régimes laminaire et transitoire :

\begin{tabular}{|c|c|c|c|}
\hline & $\begin{array}{c}\text { Débit } \\
\left.\text { (en } \mathrm{m}^{3} / \mathrm{s}\right) \\
-\end{array}$ & $\begin{array}{c}\text { Vitesse } \\
\text { moyenne } \\
\bar{V}(\text { en m/s) } \\
-\end{array}$ & $\begin{array}{c}\text { Nombre de } \\
\text { Reynolds } \\
\text { Re } \\
-\end{array}$ \\
\hline Ecoulement no 1 & $0,557 \times 10^{-4}$ & 0,71 & 1770 \\
\hline Ecoulement no 2 & $0,691 \times 10^{-4}$ & 0,88 & 2210 \\
\hline Ecoulement no 3 & $0,880 \times 10^{-4}$ & 1,12 & 2790 \\
\hline Ecoulement no 4 & $1,005 \times 10^{-4}$ & 1,28 & 3200 \\
\hline Ecoulement no 5 & $1,115 \times 10^{-4}$ & 1,42 & 3540 \\
\hline
\end{tabular}

Remarques. - Sur notre installation, il ne nous a pas été possible :

- de travailler avec des nombres de Reynolds inférieurs à 1770 en raison du phénomène de sédimentation des particules, trop sensible aux faibles débits ;

- d'atteindre des nombres de Reynolds situés en régime turbulent, limités que nous étions par les possibilités de l'installation.

5. Choix des modèles analytiques. Exploitation des données. - La théorie de Roevros, brièvement exposée précédemment, ne donne pas des modèles compatibles avec les spectres expérimentaux. Nous nous sommes donc dirigés vers une exploitation semiempirique des résultats.

5.1 CHOIX DES MODÈLES. - Parmi les expressions analytiques, nous avons envisagé de retenir celles qui conduisent à des relations facilement utilisables entre les grandeurs caractéristiques des spectres et celles des écoulements. Les modèles que nous proposons ci-dessous répondent à ce critère.

a) Modèle M 1 (Fig. 6a). - Le premier modèle de spectre choisi est défini par :

$$
G^{[1]}(v)=\beta \cdot v^{a} \cdot \exp (-v / b)
$$

$a$ et $b$ sont des paramètres qui permettent d'ajuster $G^{[1]}(v)$ à la forme du spectre expérimental $G(v)$.

Imposer la coïncidence des maxima $\left(v_{\max }\right)$ des deux spectres et celle des points à mi-hauteur $\left(v_{\mathrm{c}}\right)$,
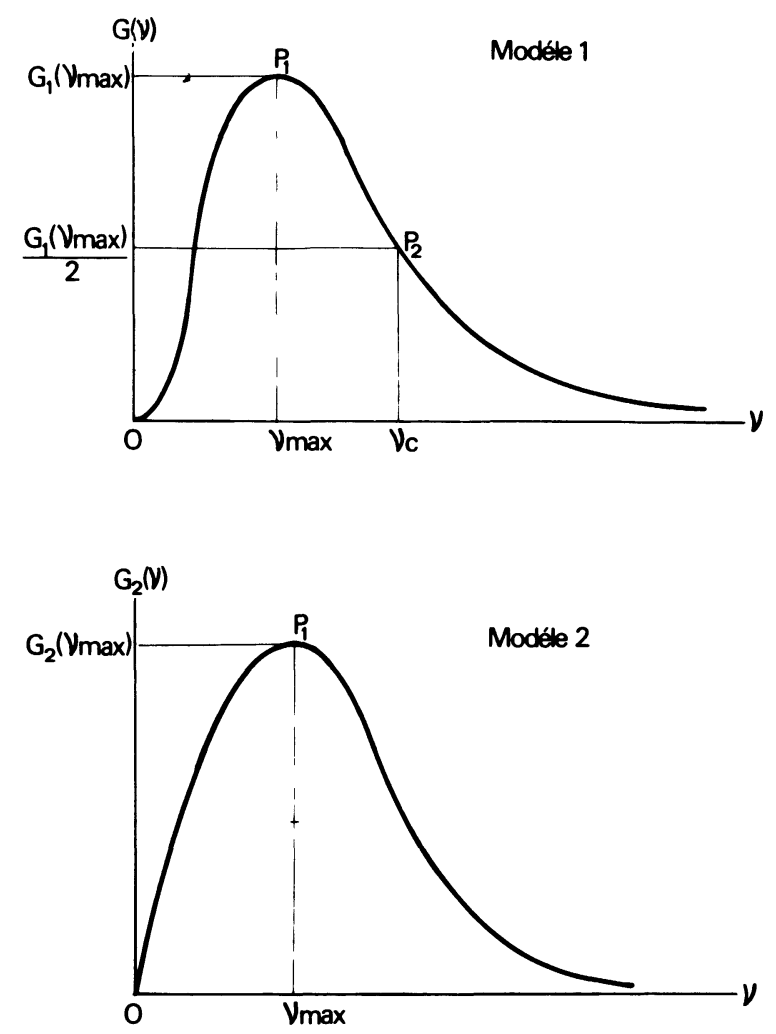

Fig. 6. - Définition des modèles de spectres théoriques.

[Theoretical models of spectra.]

situés à droite du maximum, permet de déduire la valeur des paramètres :

$$
a=\frac{\operatorname{Ln} 2}{(1 / g)-1+\operatorname{Ln} g} ; \quad b=\frac{v_{\max }[(1 / g)-1+\operatorname{Ln} g]}{\operatorname{Ln} 2}
$$

avec

$$
g=\frac{v_{\max }}{v_{\mathrm{c}}}
$$

$a$ et $b$ étant liés par la relation $a b=v_{\max }$.

Le paramètre de forme $g$ est compris entre 0 et 1 puisque, en théorie, $v_{\mathrm{c}}$ peut varier entre $v_{\max }$ et l'infini.

Le graphe de la figure 7 permet d'obtenir directement à partir du paramètre de forme, déduit des spectres expérimentaux, la valeur du paramètre $a$ puis du paramètre $b$ de la loi théorique (1). Le calcul du coefficient $\beta$ conduit à :

$$
\beta=G_{\max }\left(e / v_{\max }\right)^{a} .
$$

L'expression $W^{[1]}$ de l'énergie du signal de réponse Doppler de l'écoulement s'établit à partir du théorème de Parseval [9] :

$$
W^{[1]}=2 \int_{0}^{+\infty} G^{[1]}(v)^{2} \cdot \mathrm{d} v .
$$

Si nous effectuons le changement de variable $x=2 \cdot v / b$, nous obtenons la relation :

$$
W^{[1]}=2^{-2 a} \cdot \beta^{2} \cdot b^{(2 a+1)} \int_{0}^{+\infty} x^{2 a} \cdot \exp (-x) \cdot \mathrm{d} x .
$$




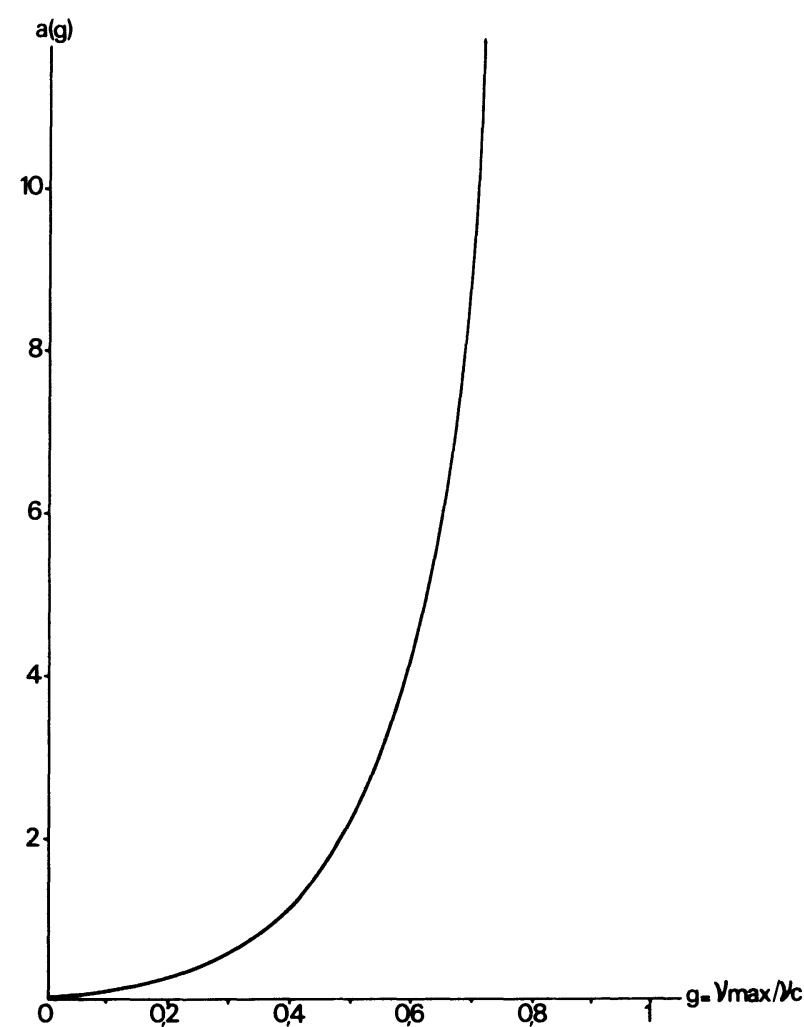

Fig. 7. - Variation du coefficient (a) avec le paramètre de forme ( $q$ ) [Relationship between coefficient $(a)$ and shape parameter $(g)$.]

Dans l'intégrale apparaît l'expression, correspondant à $n=2 a+1$, de la fonction $\Gamma(n)$ égale à :

$$
\Gamma(n)=\int_{0}^{+\infty} x^{(n-1)} \cdot \exp (-x) \cdot \mathrm{d} x .
$$

D'où :

$$
W^{[1]}=2^{-2 a} \cdot \beta^{2} \cdot b^{(2 a+1)} \cdot \Gamma(2 a+1) .
$$

Si nous remplaçons $b$ et $\beta$ par leurs valeurs précédemment calculées, et si nous posons :

$$
\dot{K(g)}=(e / 2 a)^{2 a} . \Gamma(2 a)
$$

nous obtenons :

$$
W^{[1]}=2 . G^{2}\left(v_{\max }\right) \cdot v_{\max } \cdot K(g) .
$$

Si nous connaissons la fonction $K(g)$, représentée sur la figure 8, il est alors possible de déterminer l'énergie $W^{[1]} \mathrm{du}$ signal de réponse Doppler de l'écoulement.

Expression de la vitesse moyenne de l'écoulement. A partir de la relation donnée par Roevros :

$$
\bar{v}=\alpha \cdot \bar{V}=\int_{0}^{+\infty} v \cdot G^{2}(v) \cdot \mathrm{d} v / \int_{0}^{+\infty} G^{2}(v) \cdot \mathrm{d} v
$$

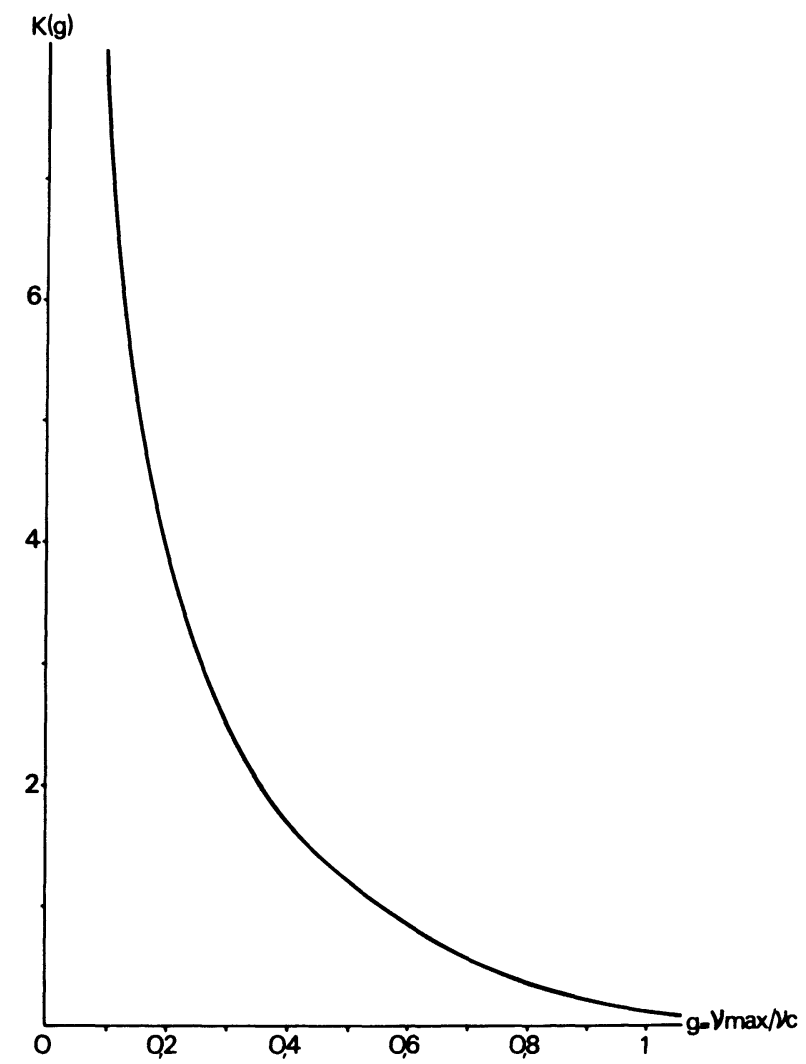

Fig. 8. - Evolution de la fonction $(K)$ avec le paramètre de forme $(g)$.

[Relationship between function $(K)$ and shape parameter $(g)$.]

nous pouvons, pour le modèle de spectre adopté, exprimer la vitesse moyenne sous la forme :

$$
\begin{aligned}
\bar{V}^{[1]} & =\frac{1}{\alpha} \cdot \frac{\int_{0}^{+\infty} v^{(2 a+1)} \cdot \exp (-2 v / b) \cdot \mathrm{d} v}{\int_{0}^{+\infty} v^{2 a} \cdot \exp (-2 v / b) \cdot \mathrm{d} v} \\
& =\frac{v_{\max }}{2 \cdot \alpha \cdot a} \cdot \frac{\Gamma(2 a+2)}{\Gamma(2 a+1)}
\end{aligned}
$$

L'utilisation de la relation de récurrence entre les fonctions $\Gamma$ conduit à la relation :

$$
\bar{V}^{[1]}=\frac{v_{\max }}{\alpha}\left(1+\frac{1}{2 a}\right) .
$$

b) Modèle M2 (Fig. 6b). - L'expression mathématique du modèle proposé s'identifie à celle de la loi de probabilité de Rayleigh. Elle s'écrit :

$$
G^{[2]}(v)=\gamma \cdot \frac{v}{q^{2}} \cdot \exp \left(-v^{2} / 2 q^{2}\right) .
$$

Les paramètres $\gamma$ et $q$ se déterminent en écrivant la coïncidence du maximum $G^{[2]}\left(v_{\max }\right)$ avec son homo- 
logue $G\left(v_{\max }\right)$ sur le spectre expérimental. Nous obtenons les expressions :

$$
\gamma=G\left(v_{\max }\right) \cdot v_{\max } \cdot \exp (1 / 2) \text { et } q=v_{\max } .
$$

Le calcul de l'énergie $W^{[2]}$ du signal de réponse Doppler de l'écoulement conduit à :

$$
W^{[2]}=e . \Gamma(3 / 2) \cdot v_{\max } \cdot G^{2}\left(v_{\max }\right) .
$$

Comme pour le modèle M1, la relation (2) permet de déduire l'expression de la vitesse moyenne de l'écoulement :

$$
\bar{V}^{[2]}=v_{\max } / \alpha \cdot \Gamma(3 / 2) .
$$

Il est important de noter que les relations de Parseval (donnant l'énergie $W$ ) et de Roevros (concernant la vitesse moyenne $\bar{V}$ ) n'imposent aucune condition sur le profil de vitesses de l'écoulement à l'intérieur de la conduite.

c) Utilisation conjuguée des modèles 1 et 2 . $\mathrm{La}$ confrontation avec l'expérience montre que le modèle M1, s'il décrit assez bien la partie du spectre située à gauche du maximum, ne s'accorde qu'imparfaitement avec la partie située à droite. Par contre, le modèle M2 lisse le spectre d'une façon inverse. Aussi, l'utilisation plus rationnelle de ces modèles permet d'obtenir une meilleure représentation des spectres. Nous pourrons donc utiliser le modèle M2 pour représenter la partie des spectres située à droite du maximum $v_{\max }$ et le modèle M1 pour la partie gauche. Nous appellerons ce modèle $\mathrm{M}[1+2]$.

L'expression de l'énergie de réponse Doppler se met alors sous la forme :

$$
W^{[1+2]}=\int_{0}^{v_{\max }} \beta^{2} \cdot v^{2 a} \cdot \exp (-2 v / b) \cdot \mathrm{d} v+\int_{v_{\max }}^{+\infty} \gamma^{2} \cdot \frac{v^{2}}{q^{4}} \cdot \exp \left(-v^{2} / q^{2}\right) \cdot \mathrm{d} v .
$$

Chaque intégrale peut s'exprimer au moyen de la fonction Gamma incomplète notée $\Gamma^{*}$ :

$$
\Gamma^{*}(s, z)=\frac{z^{-s}}{\Gamma(s)} \cdot \int_{0}^{z} \mathrm{e}^{-u} \cdot u^{(s-1)} \cdot \mathrm{d} u
$$

dont les valeurs [13] permettent le calcul des intégrales de la relation $W^{[1+2]}$. Nous obtenons :

- Pour la première intégrale notée $W_{1}^{[1+2]}$ :

$$
W_{1}^{[1+2]}=\left[2 \cdot G^{2}\left(v_{\max }\right) \cdot v_{\max } \cdot K(g)\right] \cdot v_{\max }^{(2 a+1)} \cdot \Gamma^{*}\left(2 a+1, v_{\max }\right) .
$$

Dans le terme entre crochets, nous reconnaissons l'expression de l'énergie $W^{[1]}$ correspondant au modèle M1.

- Pour la deuxième intégrale notée $W_{2}^{[1+2]}$ :

$$
W_{2}^{[1+2]}=\left[e \cdot \Gamma(3 / 2) \cdot v_{\max } \cdot G^{2}\left(v_{\max }\right)\right] \cdot\left(1-v_{\max }^{3 / 2} \cdot \Gamma^{*}\left(3 / 2, v_{\max }\right)\right) .
$$

L'expression entre crochets représente l'énergie $W^{[2]}$ calculée dans le modèle M2.

L'énergie $W^{[1+2]}$ du signal de réponse Doppler s'écrit alors :

$$
W^{[1+2]}=W^{[1]} \cdot v_{\max }^{(2 a+1)} \cdot \Gamma^{*}\left(2 a+1, v_{\max }\right)+W^{[2]} \cdot\left(1-v_{\max }^{3 / 2} \cdot \Gamma^{*}\left(3 / 2, v_{\max }\right)\right) .
$$

Dans le cas de cette double représentation spectrale, la relation donnant la vitesse moyenne devient :

$$
\bar{V}^{[1+2]}=\frac{\bar{V}^{[1]} \cdot W^{[1]} \cdot v_{\max }^{(2 a+2)} \cdot \Gamma^{*}\left(2 a+2, v_{\max }\right)+\bar{V}^{[2]} \cdot W^{[2]} \cdot\left(1-v_{\max }^{2} \cdot \Gamma^{*}\left(2, v_{\max }\right)\right)}{W^{[1]} \cdot v_{\max }^{(2 a+1)} \cdot \Gamma^{*}\left(2 a+1, v_{\max }\right)+W^{[2]} \cdot\left(1-v_{\max }^{2} \cdot \Gamma^{*}\left(3 / 2, v_{\max }\right)\right)}
$$

qui peut s'écrire sous la forme simplifiée :

$$
\bar{V}^{[1+2]} \# \frac{\bar{V}^{[1]} W_{1}^{[1+2]}+\bar{V}^{[2]} \cdot W_{2}^{[1+2]}}{W_{1}^{[1+2]}+W_{2}^{[1+2]}} .
$$


6. Application des modèles aux spectres expérimentaux. - Considérons, par exemple, figure 9 le spectre d'amplitude du signal de réponse Doppler de l'écoulement correspondant au nombre de Reynolds de 1790.

Sur la figure 10 la courbe $C_{\mathrm{sm}}$ montre le tracé moyen $\mathrm{du}$ spectre expérimental dont les caractéristiques spectrales sont :

- fréquence du maximum : $v_{\max }=1590 \mathrm{~Hz}$,

- fréquence d'étalement : $v_{\mathrm{c}}=2540 \mathrm{~Hz}$,

- amplitude du maximum : $G_{\max }=0,173 \mathrm{~V}$.

La formulation des expressions mathématiques des modèles est alors :

Modèle $1: G^{[1]}(v)=3,10098 \cdot v^{5,382} \cdot \exp (-v / 0,295)$

Modèle $2: G^{[2]}(v)=0,17939 \cdot v \cdot \exp (-v / 5,056)$.

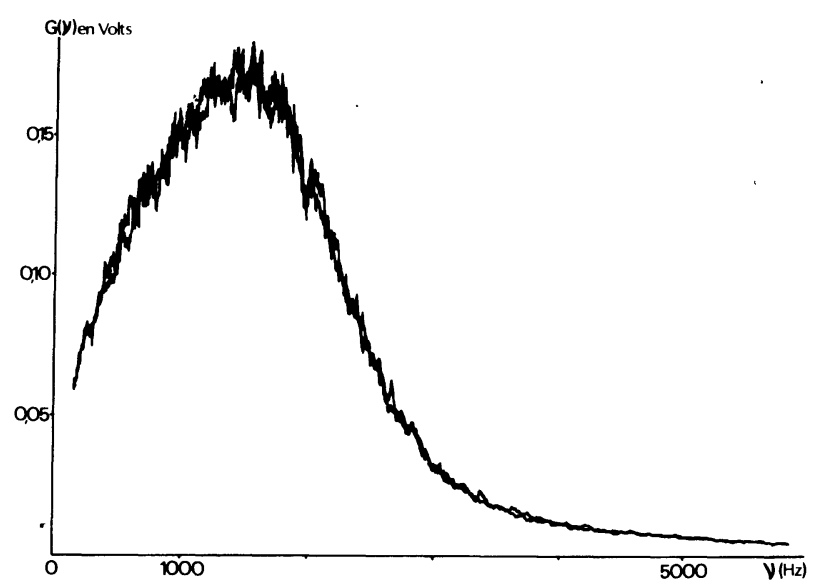

Fig. 9. - Spectre de réponse Doppler de l'écoulement correspondant à $\operatorname{Re}=2790$, pour $\theta_{\mathrm{E}}=70^{\circ}$ et $\theta_{\mathrm{R}}=55^{\circ}$.

[Doppler spectrum of the flow corresponding to $R e=2790$, for $\theta_{\mathrm{E}}=70^{\circ}$ and $\theta_{\mathrm{R}}=55^{\circ}$.]

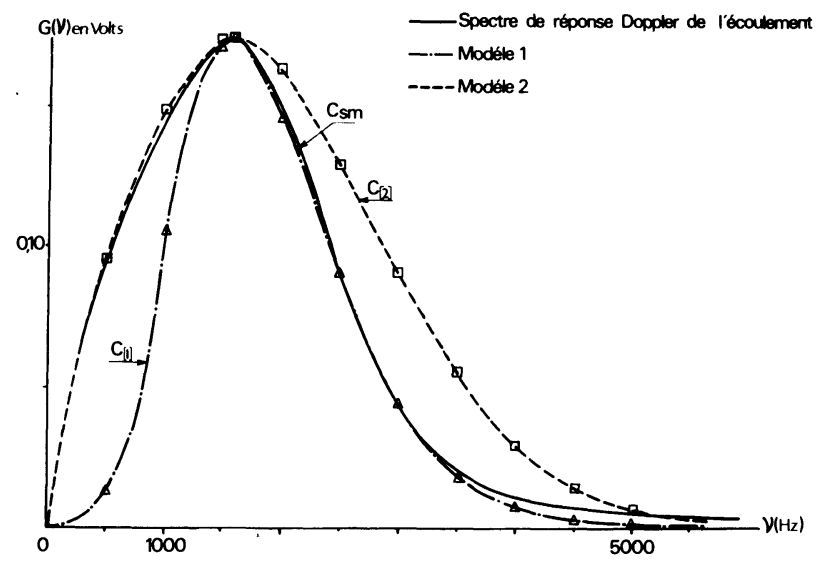

Fig. 10. - Comparaison des modèles avec le spectre de réponse Doppler de l'écoulement correspondant à $\operatorname{Re}=2790$.

[Comparison of the models with the Doppler spectrum corresponding to $R e=2790$.]
Les graphes correspondant à ces modèles sont représentés sur la figure 10 , respectivement par les courbes $C^{[1]}$ et $C^{[2]}$.

Le tableau qui suit rassemble les résultats obtenus sur 5 écoulements étudiés.

Précision des résultats. - La comparaison des valeurs déduites des expressions mathématiques avec celles données par l'expérimentation, est effectuée à partir du calcul des erreurs relatives données par l'expression :

$$
\chi^{[\mathrm{M}]}=\frac{\bar{V}^{[\mathrm{M}]}-\bar{V}}{\bar{V}}=\frac{R e^{[\mathrm{M}]}-R e}{\operatorname{Re}} .
$$

7. Conclusions. - Le problème de l'étude ultrasonore des écoulements permanents de fluides, chargés en particules, a été abordé par le biais des spectres de fréquences des signaux de réponse Doppler des écoulements correspondants. Ces spectres permettent d'obtenir des renseignements interprétables dans le cadre d'une recherche des paramètres caractéristiques de tels écoulements.

La modélisation des spectres expérimentaux permet d'établir des relations entre ces paramètres et certaines grandeurs spectrales comme la fréquence et l'amplitude du maximum et une fréquence d'étalement du spectre. Trois modèles ont été établis. Pour deux d'entre eux, nous avons obtenu des relations directement exploitables qui ont conduit à la détermination de l'énergie de réponse Doppler des écoulements, des vitesses moyennes ou des nombres de Reynolds. Les écarts enregistrés sur les grandeurs précédentes sont, par rapport à leurs valeurs expérimentales, de l'ordre ou inférieurs à $5 \%$. Le troisième modèle, construit à partir des deux premiers, entraîne un lissage plus précis du tracé des spectres et donc une meilleure évaluation de l'énergie. Son utilisation, dans la procédure de calcul de la vitesse moyenne ou du nombre de Reynolds, conduit à une erreur relative comparable à celles enregistrées avec les précédents modèles. Par conséquent, au vu des résultats consignés dans le tableau seuls les modèles M1 ou M2 suffiront à la détermination des paramètres de l'écoulement.

Cette méthode d'étude des écoulements de suspension ouvre des possibilités d'application à l'étude écoulements d'autres dispersions comme les émulsions, les bouillons ou les mousses, et à l'étude de l'écoulement sanguin en hémodynamique.

Sur le plan technologique lié à celui de l'instrumentation biomédicale, nous cherchons à réaliser un débitmètre ultrasonore équipé d'un analyseur sommaire (géré par microprocesseur) qui fournira, par affichage, les paramètres dynamiques de l'écoulement que nous avons mis en évidence dans cette étude. Ceux-ci devraient permettre aux praticiens de disposer d'un appareillage plus rationnel leur permettant d'élaborer des diagnostics tendant vers une précision accrue. 


\begin{tabular}{|c|c|c|c|c|c|}
\hline & & $\begin{array}{c}\text { Energie du Signal } \\
\text { de réponse Doppler } \\
\qquad W\end{array}$ & $\begin{array}{l}\text { Vitesse moyenne } \\
\text { de l'écoulement } \\
\bar{V}(\text { en } \mathrm{m} / \mathrm{s})\end{array}$ & $\begin{array}{l}\text { Nombre de } \\
\text { Reynolds } \\
\text { Re }\end{array}$ & $\chi(\mathrm{en} \%)$ \\
\hline \multirow{5}{*}{$\begin{array}{l}\text { Ecoulement } \\
\quad n^{\circ} 1\end{array}$} & & - & - & - & - \\
\hline & Expérience & & $\underline{0,71}$ & 1770 & \\
\hline & M1 & 0,057 & $\overline{0,73}$ & $\overline{1800}$ & 2 \\
\hline & M2 & 0,082 & 0,75 & 1850 & 4,5 \\
\hline & $\mathrm{M}(1+2)$ & 0,071 & 0,74 & 1925 & 3 \\
\hline \multirow{4}{*}{$\begin{array}{l}\text { Ecoulement } \\
\quad n^{0} 2\end{array}$} & Expérience & & 0,88 & 2210 & \\
\hline & M1 & 0,068 & 0,90 & 2250 & 2 \\
\hline & M2 & 0,104 & 0,91 & 2275 & 3 \\
\hline & $M(1+2)$ & 0,091 & 0,90 & 2250 & 2 \\
\hline \multirow{4}{*}{$\begin{array}{l}\text { Ecoulement } \\
\text { no } 3\end{array}$} & Expérience & & 1,12 & 2790 & \\
\hline & M1 & 0,073 & $\frac{1,12}{1,13}$ & 1815 & 1 \\
\hline & M2 & 0,115 & 1,16 & 2905 & 4 \\
\hline & $\mathrm{M}(1+2)$ & 0,094 & 1,14 & 2850 & 2 \\
\hline \multirow{4}{*}{$\begin{array}{l}\text { Ecoulement } \\
\text { no } 4\end{array}$} & Expérience & & 1,28 & 3200 & \\
\hline & M1 & 0,075 & $\frac{, 33}{1,33}$ & 3325 & 4 \\
\hline & M2 & 0,111 & 1,32 & 3300 & 3 \\
\hline & $\mathrm{M}(1+2)$ & 0,096 & 1,32 & 3300 & 3 \\
\hline \multirow{4}{*}{$\begin{array}{l}\text { Ecoulement } \\
\text { no } 5\end{array}$} & Expérience & & 1,42 & 3540 & \\
\hline & M1 & 0,072 & $\frac{1,2}{1,50}$ & 3750 & 6 \\
\hline & M2 & 0,113 & 1,49 & 3725 & 5 \\
\hline & $\mathrm{M}(1+2)$ & 0,093 & 1,48 & 3700 & 4,5 \\
\hline
\end{tabular}

Bibliographie

[1] Peronneau, P., Hinglais, J., Pellet, M. et Leger, F., Vélocimètre sanguin, par effet Doppler, à émission ultrasonore pulsée, Onde Electr. 50 (1970) 369-389.

[2] Flax, S. W., Webster, J. G. et Updike, S. J., Noise and functional limitations of the Doppler blood flowmeter, cardiovascular applications of ultrasound, edited by Robert S. Reneman (American Elsevier, Publishing Company Inc. New York) 1974, 18-31.

[3] Bournat, J. P., Mesure des fréquences Doppler, vélocimétrie ultrasonore Doppler, ed. Peronneau P. (INSERM, Paris) Vol. 34 (1974) p. 123-145.

[4] Vera, J. C. et Lefort, M. F., Troisième Congrès de Mécanique de l'A.U.M., Grenoble, 1977.

[5] Vera, J. C., Thèse de Docteur-Ingénieur, Nancy, 1977.

[6] Vera, J. C., Lefort, M. F., L'Huillier, J. P. et Stoltz, J. F., Contribution à l'étude du débit sanguin par analyse spectrale basses fréquences des signaux Doppler ultrasonores. Application à un modèle d'écoulement permanent, Biorheology 15 (1978) 181-191.

[7] GreEn, P. S., Spectral broadening of acoustic reverberation in Doppler shift fluid flowmeters, J. Acoust. Soc. Am. 36 (1964) 1383-1390.
[8] Edwards, R. V., Angus, J. C., French, M. J. et Dunning, J. W., Spectral analysis of the signal from the laser flowmeter : time-independent systems, J. Appl. Phys. 2 (1971) 837-850.

[9] CARPentier, M. H., La théorie du signal et son application au radar, vélocimétrie ultrasonore Doppler, ed. Peronneau P. (INSERM, Paris) 34 (1974) 31-67.

[10] Roevros, J. M. J. G., Analog processing of C. W. Doppler flowmeter signal to determine average frequency shift momentaneously without the use of a wave analyser, cardiovascular applications of ultrasound, edited by Robert S. Reneman (American Elsevier Publishing Company Inc., New York) 1974, p. 43-54.

[11] Schlichting, H., Boundary-layer theory (Mc Graw-Hill Book Company, New York) 1968

[12] Gosling, R. G., KING, D. M., Continuous wave ultrasound as an alternative and complement to $X$-rays in vascular examinations, cardiovascular applications of ultrasound, edited by Robert Reneman (American Elsevier, Publishing Company Inc., New York) 1974, p. 266-282.

[13] Handbook of mathematical functions (Dover Publ. Inc., New York) 1965. 